$$
\text { Pontifícia Universidade C Católica }
$$

Sílvia Beatriz Alexandra Becher-Costa

\title{
O Significado da Mudança ou a Mudança de Significado? Análise da Implantação de Modelos de Gestão de Pessoas por Competências
}

Tese de Doutorado

Tese apresentada ao programa de Pós-Graduação em Administração do Departamento de Administração da PUC-Rio como parte dos requisitos parciais para obtenção do título de Doutor em Administração.

Orientadora: Profa. Patrícia Amélia Tomei 


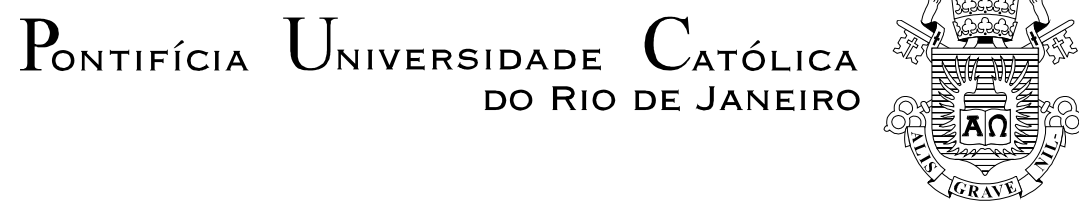

Silvia Beatriz Alexandra Becher-Costa

\begin{abstract}
O Significado da Mudança ou a Mudança de Significado? Análise da Implantação de Modelos de Gestão de Pessoas por Competências
\end{abstract}

Tese apresentada como requisito parcial para obtenção do grau de Doutor pelo Programa de Pós-graduação em Administração de Empresas da PUC-Rio. Aprovada pela Comissão Examinadora abaixo assinada.

Profa. Patrícia Amélia Tomei Orientadora Departamento de Administração - PUC-Rio

Prof. José Roberto Gomes da Silva Departamento de Administração - PUC-Rio

Profa. Maria do Carmo Leite de Oliveira Departamento de Letras - PUC-Rio

Profa. Adriana Victoria Garibaldi de Hilal COPPEAD - UFRJ

Prof. Jorge Augusto de Sá Brito e Freitas Universidade Estácio de Sá

Prof. Paulo Cesar Teixeira Departamento de Administração - PUC-Rio

Prof. João Pontes Nogueira Vice-Decano de Pós-Graduação do CCS

Rio de Janeiro, 11 de abril de 2006 
Todos os direitos reservados. É proibida a reprodução total ou parcial do trabalho sem autorização da universidade, da autora e da orientadora.

\title{
Sílvia Beatriz Alexandra Becher-Costa
}

Graduou-se em Letras (Português- Inglês) pela PUC-Rio, tendo concluído a Licenciatura em 1976 e o Bacharelado com habilitação em Tradução e Interpretação em 1977. Em 1980, concluiu Mestrado em Letras na PUC-Rio, com dissertação sobre Formas de Polidez em Inglês e Português. Tem atuado como professora em diversas instituições de ensino, a saber: PUC-Rio (Departamento de Letras); UFRJ (Faculdade de Letras) e Colégio Pedro II, ministrando cursos na área de língua inglesa, metodologias de ensino de línguas estrangeiras, tradução e interpretação simultânea. Dentre as várias funções que exerceu, destacam-se os cargos administrativos ocupados em grandes instituições de ensino, tais como Subsecretária de Planejamento do Colégio Pedro II, Coordenadora de Graduação (Letras / PUC-Rio). Atualmente faz o planejamento e a coordenação administrativa dos Cursos de Extensão em Letras e do IPEL (Instituto de Pesquisa e Ensino de Línguas) na PUCRio. Atua como intérprete de conferências. Tem publicações com temáticas da área de ensino de línguas e de administração.

Ficha Catalográfica

\begin{abstract}
Becher-Costa, Sílvia Beatriz Alexandra
O significado da mudança ou a mudança do significado? Análise da implantação de modelos de gestão de pessoas por competências / Sílvia Beatriz Alexandra Becher-Costa ; orientadora: Patrícia Amélia Tomei . - Rio de Janeiro : PUC, Departamento de Administração, 2006.
\end{abstract}

$388 \mathrm{f.} \mathrm{;} 30 \mathrm{~cm}$

Tese (doutorado) - Pontifícia Universidade Católica do Rio de Janeiro, Departamento de Administração

Inclui bibliografia.

1. Administração - Teses. 2. Gestão por competências. 3. Mudança organizacional. 4. Processo de comunicação. 5. Construção de significado. I. Tomei, Patrícia Amélia. II. Pontifícia Universidade Católica do Rio de Janeiro. Departamento de Administração . III. Título. 
É com muito amor e saudade que dedico esta tese aos que deixaram um grande vácuo no meu coração.

A minha mãe, por ter sido tanto, sempre, e ter me ensinado a buscar sempre o melhor.

Ao meu filho Guilherme, por ter sido um grande menino e pelo que poderia ter sido. 
Agradecimentos MUITO especiais

Ao meu querido filho Daniel e meu marido Heitor,

pelas inúmeras demonstrações de carinho, tolerância e apoio ao longo desta longa e, muitas vezes, árdua caminhada. Com amor souberam entender todas as minhas ausências. 


\section{Agradecimentos}

À Patrícia Tomei, orientadora e amiga, por toda confiança, inspiração e suporte.

Aos queridos amigos e 'chefes', Marco Antonio Brandão Fernandes e Maria Helena Soares Sampaio, pelo apoio e incentivo que deram.

Aos colegas de percurso, Kátia Rousseau, Marcos Cohen e Renata Buarque Coutinho, companheiros do Doutorado de Administração, pelos muitos momentos em que tanto compartilhamos. E à Lourdes Sette, que traçou caminho paralelo no Doutorado de Letras, pelas horas de interlocução.

Aos professores do programa de Pós-Graduação do Departamento de Administração, pelas lições e exemplos, em especial: Paulo César Motta, Diana Macedo-Soares, Antonio Carlos Figueiredo, Sérgio Proença Leitão, Jorge Ferreira da Silva e Hélène Bertrand.

Aos tantos amigos e colegas de trabalho que serviram de escuta às minhas aflições e conquistas e que tinham sempre uma palavra de estímulo. É impossível listar todos, mas merecem destaque: Esther Ozon Monfort, Polyana Wallier, Lucia Pacheco de Oliveira e Tania Saliés.

Aos meus queridos familiares próximos, pelos inúmeros "não posso" que ouviram e compreenderam. Lea, Roberto e D. Laura, prometo compensar todas as faltas!

Aos alunos Pedro Brum Junior, Roberta Moura Leopoldino, José Mauro Ferreira Pinheiro, Juliana Roiz, pelo muito que ajudaram com as transcrições, e, especialmente, a Rafaela Zukier e Cristianne Prescilla da Paz e Silva, que além de serem um grande auxílio com mais transcrições ainda estavam sempre carinhosamente me instigando a prosseguir.

Aos entrevistados nas empresas pesquisadas que me receberam com tanto entusiasmo e atenção. 


\section{Resumo}

Becher-Costa, Sílvia Beatriz Alexandra; Tomei, Patrícia Amélia (Orientadora). O Significado da Mudança ou a Mudança de Significado? Análise da Implantação de Modelos de Gestão de Pessoas por Competências. Rio de Janeiro, 2006. 388p. Tese de Doutorado Departamento de Administração, Pontifícia Universidade Católica do Rio de Janeiro.

As organizações estão enfrentando um ambiente turbulento que exige mudanças rápidas e paradigmáticas. Para atender a estas demandas, modelos de gestão de pessoas por competências têm sido adotados como resposta à necessidade de um maior envolvimento dos indivíduos e busca de diferenciais competitivos para a organização (Dutra, 2004). Esta pesquisa teve como objetivo analisar a implantação destes modelos de gestão de pessoas com base em competências e os significados sinalizados pelas iniciativas de comunicação desta mudança organizacional, a partir da ótica de diferentes modelos referenciais (Weick, 1999; Klein, 1996; Ford \& Ford, 1995; Van de Ven \& Poole, 1995; Lewin, 1951). Utilizou-se uma abordagem qualitativa, com múltiplos estudos de caso. Foram selecionadas sete empresas de um setor da economia brasileira escolhido intencionalmente devido à intensidade e complexidade de seu processo de mudança: o setor de telecomunicações. Os dados foram coletados por meio de investigação documental, entrevistas semi-estruturadas e questionários fechados. As conclusões da pesquisa ressaltam a importância da comunicação na instauração do projeto de mudança e apontam para a necessidade de atentar para os significados que são construídos na organização como desdobramento dos processos de divulgação da mudança planejada e das diferentes fases de implantação dos modelos de gestão de pessoas com base em competências.

\section{Palavras-chave}

Gestão por competências; mudança organizacional; processo de comunicação; construção de significado. 


\section{Abstract}

Becher-Costa, Sílvia Beatriz Alexandra; Tomei, Patrícia Amélia (Advisor). The Meaning of Change or the Change of Meaning? An Analysis on Implementing Competency-Based Models in People Management Rio de Janeiro, 2006. 388p. PhD Dissertation Departamento de Administração, Pontifícia Universidade Católica do Rio de Janeiro.

Today's turbulent business environment demands speedy and paradigmatic changes. To meet these challenges, organizations are adopting competency-based people-management models as a response to the need for greater individual commitment and as an important strategy for gaining a competitive edge (Dutra, 2004). This study analyzes the implementation of such competency-based models and their meaning for organizational change, as signaled by the communication initiatives the companies adopted, from the perspective of different theoretical frameworks (Weick, 1999; Klein, 1996; Ford \& Ford, 1995; Van de Ven \& Poole, 1995; Lewin, 1951). Using a qualitative approach, multiple case studies were discussed. Seven companies from the telecommunications sector were selected, as representative of a high clock speed industry that in Brazil continuously undergoes intense and complex changes. The research findings are based on information collected from in-depth semi-structured interviews, closed-answer questionnaires and archival data. The findings reinforce the importance of communication efforts for putting a change project into effect. They also highlight the need to unravel the meanings triggered by information strategies used during the different phases of project implementation considering the competency-based HR management programs as examples of planned change.

\section{Keywords}

Competency-based management; organizational change; communication; constructing meaning. 


\section{Sumário}

1. Introdução 16

1.1. A Situação-problema 16

1.2. Objetivos 23

1.2.1. Objetivo Principal 24

$\begin{array}{ll}\text { 1.2.2. Objetivos intermediários } & 24\end{array}$

1.3. Premissas e Suposições $\quad 27$

1.4. Delimitação do Estudo $\quad 29$

1.5. Relevância do Estudo 31

2. Referencial Teórico $\quad 34$

2.1. Entendendo a Mudança Organizacional 35

2.1.1. Introdução ao tema de mudança organizacional 35

2.1.2. Mudança Organizacional e o Contexto 37

2.1.3. O Processo de Mudança Organizacional 39

2.1.4. Propriedades da Mudança Organizacional 41

2.1.4.1. O Desenvolvimento da Mudança 45

2.1.4.2. Taxionomias da Mudança 46

2.1.5. A Mudança Organizacional e os Indivíduos 49

2.1.5.1. Reações à Mudança 49

2.1.5.2. Estratégias de Enfrentamento de Mudanças 51

2.1.5.3. Emoções evocam ações no processo da mudança organizacional 54

2.2. Construindo Significados em Projetos de Mudanças 58

2.2.1. Como entender o termo 'comunicação'?

2.2.2. O papel da comunicação na mudança 59

2.2.3. Domínios da comunicação na mudança 61

2.2.4. Estratégias de comunicação na mudança 63

2.2.5. A co-construção de significados através da comunicação 67

2.2.6. A comunicação da mudança no contexto desta pesquisa 72

2.2.7. Uma mídia de destaque na comunicação: os folhetos 74

2.2.8. O panorama multi-semiótico 75

2.2.8.1. O cenário da análise multimodal 76

2.2.8.2. As metafunções da comunicação - correlações entre a comunicação verbal e a visual $\quad 78$

2.2.8.3. O sistema de relações entre texto e imagem 79

2.2.9. Papel das metáforas na produção de significado 85

2.3. Novas Formas de Gestão de Pessoas: O modelo de Gestão de Pessoas por Competências $\quad 88$

2.3.1. Origens e Histórico do Modelo de Gestão de Competências 90 
2.3.2. Concepções Variadas 92

2.3.3. O Modelo de Gestão de Pessoas por Competências 99

2.3.4. Aplicando o Modelo de Gestão por Competências nas Empresas 105

2.3.5. Riscos e Barreiras na Implantação do Sistema de Gestão de Pessoas por Competências

3. Metodologia 113

3.1. Pressupostos Epistemológicos da Pesquisa 113

3.2. Caracterização da Pesquisa 115

3.3. Seleção dos Casos 118

3.4. Coleta de Dados 121

3.4.1. As entrevistas 121

3.4.2. Os materiais de divulgação 123

$\begin{array}{ll}\text { 3.4.3. Os questionários } & 124\end{array}$

3.5. Tratamento dos Dados 125

3.6. Limitações 129

4. Caracterização d Contexto Pesquisado 131

4.1. O Setor de Telecomunicações no Brasil: O Contexto Histórico e o Mercado Atual para Telefonia Fixa e Móvel 131

4.2. As Empresas Estudadas 140

4.2.1. Panorama Geral 140

4.2.2. Breves Caracterizações 143

4.2.2.1. Caso 1: VIVO 143

4.2.2.2. Caso 2: CLARO 147

4.2.2.3. Caso 3: TIM 150

4.2.2.4. Caso 4: TELEMAR - OI 151

4.2.2.5. Caso 5: TELEFONICA EMPRESAS 155

4.2.2.6. Caso 6: EMBRATEL 158

4.2.2.8. Caso 7: BRASIL TELECOM 160

5. Projetos de Gestão de Pessoas por Competências nas Empresas

Telecom Brasileiras 164

5.1. Descritores Utilizados para Desvendar os Projetos 165

5.2. Caracterização e Especificidades dos Projetos Investigados 175

5.2.1. A Régua da VIVO 176

5.2.2. O Foco da CLARO 187

5.2.3. A Pirâmide da TIM 198

5.2.4. A Bússola da TELEMAR - OI 217

5.2.5. A Lapidação da TELEFONICA 233

5.2.6. As Trilhas da EMBRATEL 246

5.2.7. A Trajetória de Crescimento da BRASIL TELECOM 256 
6. Análise dos Casos sob a Ótica da Implantação da Mudança 277

6.1. Caracterizando as Mudanças Implantadas 277

6.2. Fatores de Sucesso e Dificuldades 282

6.2.1. Barreiras e Dificuldades Encontradas 282

6.2.2. Acertos na Execução dos Projetos 290

7. Análise dos Casos sob a Ótica da Comunicação 297

7.1. A Relevância da Comunicação para as Organizações Estudadas 298

7.2. Características da Comunicação nos Casos Estudados 302

7.2.1. A Comunicação na VIVO 302

7.2.2. A Comunicação na CLARO 304

7.2.3. A Comunicação na TIM 309

7.2.4. A Comunicação na TELEMAR - OI 313

7.2.5. A Comunicação na TELEFONICA EMPRESAS 316

7.2.6. A Comunicação na EMBRATEL 319

7.2.7. A Comunicação na BRASIL TELECOM 323

7.3. Fatores Favoráveis e Desfavoráveis na Comunicação como Indicativos para o Sucesso na Implantação dos Projetos de Gestão por Competências 327

7.3.1. Classificação dos Fatores por Movimentos de Embalagem 329

7.3.2. Classificação dos Fatores por Movimentos de Agregação 333

7.3.3. Identificação de Fatores Desfavoráveis a Evitar 336

8. Conclusões 340

8.1. Conclusões Relativas à Natureza do Projeto de Gestão por Competências 342

8.2. Conclusões Relativas à Natureza da Comunicação na Implantação do Projeto de Gestão por Competências 350

9. Limitações e Recomendações 357

9.1. Limitações da Pesquisa 357

9.2. Sugestões de Novas Perspectivas para Pesquisa 359

10. Referências bibliográficas 363

$\begin{array}{ll}\text { Anexo I } & 381\end{array}$ 


\section{Lista de Figuras}

Figura 2.1. Representação das etapas de mudança na fita de Möbius 41

Figura 2.2. Perspectivas de Análise de Mudança Organizacional:

Figura 2.3 Teorias do Processo de Desenvolvimento e Mudança Organizacional 45

Figura 2.4. Síntese dos Critérios de Classificação Observados na Literatura sobre Mudança

Figura 2.5. Etapas de Mudança Organizacional: objetivos, atividades e necessidades comunicativas

Figura 2.6. Relação dos Princípios de Comunicação às Etapas de Mudança organizacional

Figura 2.7. Esquema da Análise das Estratégias Empregas para Comunicação da Mudança

Figura 2.8. Relação de Status entre Texto e Imagem 81

Figura 2.9. Relações Lógico-semânticas entre Texto e Imagem 82

Figura 2.10. Conceitos Gerais para Análise Multimodal dos Materiais

Figura 2.11. Mudanças da Gestão de Recursos Humanos $\quad 89$

Figura 2.12. O Conceito de Competência para Diferentes Autores 94

Figura 2.13. O Conceito de Competência Individual 96

Figura 2.14. Variáveis Diferenciadoras de Níveis de Complexidade 98

Figura 2.15. Conceitos do Modelo de Gestão de Pessoas Baseado em Competências de Dutra

Figura 2.16. Modelo de Gestão de Desempenho Baseado em Competências

Figura 2.17. Fases de Estruturação do Projeto de Gestão de Pessoas 108

Figura 2.18. Esquema de Colaboração para o Processo de Concepção e Implementação de Sistemas de Gestão de Pessoas

Figura 2.19. Sub-etapas da Fase 3 da Estruturação do Projeto de

Figura 3.1. Modelo de Desenvolvimento de Categorias Indutivas

$\begin{array}{ll}\text { Figura 4.1. Indicadores de Mercado } & 133\end{array}$

$\begin{array}{ll}\text { Figura 4.2. Comparativo de Receitas } & 135\end{array}$

Figura 4.3. Grupos no Mercado Brasileiro de Telecomunicações 136

Figura 4.4. Market Share de Empresas Telecom por Receita Bruta 136

Figura 4.5. Maiores Empresas em Telecomunicações no Brasil 137

\begin{tabular}{ll} 
Figura 4.6. & 137 \\
\hline & Ranking por Vendas
\end{tabular}

$\begin{array}{lll}\text { Figura 4.7. } & 138\end{array}$

$\begin{array}{lll}\text { Figura 4.8. } & \text { Ranking por receita líquida } & 138\end{array}$ 
$\begin{array}{lll}\text { Figura 4.9. } & \text { Ranking por criação de riqueza } & 138\end{array}$

$\begin{array}{lll}\text { Figura 4.10. } & 139\end{array}$

$\begin{array}{lll}\text { Figura 4.11. Maiores Empresas de Telefonia no Brasil } & 140\end{array}$

Figura 4.12. Market Share das Operadoras de Telefonia Móvel por número de celulares

Figura 4.13. Concessionárias e Empresas-Espelho 141

Figura 4.14. Market Share de Acessos em Serviços 142

Figura 4.15. Resultados de Operadoras de Telefonia Fixa 142

Figura 4.16. Estrutura Societária Básica da VIVO 143

$\begin{array}{ll}\text { Figura 4.17. Operadoras do Grupo VIVO } & 144\end{array}$

$\begin{array}{lll}\text { Figura 4.18. } & \text { Market-share da VIVO em } 2004\end{array}$

Figura 4.19. Faixa etária dos funcionários da VIVO em 2004146

$\begin{array}{lll}\text { Figura 4.20. Tempo de empresa dos funcionários da VIVO em } 2004 & 147\end{array}$

$\begin{array}{lll}\text { Figura 4.21. Área de atuação da CLARO } & 148\end{array}$

Figura 4.22. Dados operacionais da CLARO 150

Figura 4.23. Dados de Crescimento OI e VELOX em 2004

Figura 4.24. Dados de Crescimento 153

Figura 4.25. Base média de clientes da OI e índices de 'churn' 153

Figura 4.26. Distribuição de total de clientes do grupo em $2005 \quad 154$

Figura 4.27. Arquitetura da Marca Telefônica no Brasil 155

Figura 4.28. Presença do grupo Telefônica no Brasil 156

Figura 4.29. Regiões de operação da Embratel - telefonia fixa, espelho 159

Figura 4.30. Crescimento da Base de Clientes de Telefonia Móvel no ano de lançamento 161

Figura 4.31. Crescimento do Quadro de Colaboradores da BrT 162

Figura 4.32. Faixa Etária e Tempo de Serviço dos Colaboradores da Brasil Telecom 163

Figura 5.1. Macro-categorias e Descritores para o exame dos

$\begin{array}{ll}\text { projetos de gestão de pessoas por competências } & 166\end{array}$

$\begin{array}{lll}\text { Figura 5.2. } & 181\end{array}$

Figura 5.3. Distribuição Sugerida de Resultados das Equipes -

$\begin{array}{lll}\text { Figura 5.4. Organização do SAC - CLARO } & 190\end{array}$

$\begin{array}{lll}\text { Figura 5.5. O pessoal da TIM } & 202\end{array}$

Figura 5.6. Fluxo de Mecanismos de avaliação na TIM 203

Figura 5.7. Competências da TIM 209

Figura 5.8. Configuração do Modelo de Avaliação da TIM-BRASIL 216

Figura 5.9. Processo de Análise de Desempenho na TELEMAR-OI 223

Figura 5.10. Visão Geral do Processo 225

Figura 5.11. Reunião de Feedback - Fase Intermediária 226

Figura 5.12. Reunião de Feedback - Finalização do Ciclo 227

Figura 5.13. Reunião de Feedback Final. 227 
Figura 5.14. Distribuição de Peso das Metas Contratadas - TelemarOI

Figura 5.15. Entrelaçamento de Competências e Valores na Telefônica Empresas

Figura 5.16. Integração do Modelo de Gestão por Competências Telefônica Empresas

Figura 5.17. Modelo de Competências da Embratel

Figura 5.18. Competências definidas para Trajetórias de Desenvolvimento na Brasil Telecom 262

Figura 5.19. Representação do Sistema Integrado de Gestão de Pessoas - Brasil Telecom

Figura 5.20. Matriz para Diagnóstico de Desenvolvimento na Brasil Telecom

Figura 5.21. Quadro de Benefícios do Sistema Integrado Brasil Telecom

Quadro I Caracterização Geral e Macro-categoria TEMPO

Quadro II Macro-categoria DIRECIONAMENTO e ENQUADRAMENTO

$\begin{array}{lll}\text { Quadro III Macro-categoria ESTRUTURA } & 275\end{array}$

Quadro IV Macro-categoria OUTPUT 276

Figura 6.1. Objetivos e Motivações - conflitos ou coerência? 284

Figura 7.1. A Representação dos Comportamentos Requeridos - TIM 312

Figura 7.2. Dimensões da Comunicação 333

Figura 8.1. Aspectos a Serem Atendidos em Processos de Comunicação para Mudança 353 
A woman's life can really be a succession of lives, each revolving around some emotionally compelling situation or challenge, and each marked off by some intense experience. 